\title{
Flotation Froth tracking and speed measurement based on features and spatial relationship
}

\author{
Guoying ZHANG ${ }^{1, a}$, Daihong ZHOU ${ }^{1, a}$, Beihua FENG ${ }^{2}$, Wenlin GONG ${ }^{2}$ \\ ${ }^{1}$ Dept of Computer Science and Technology, China University of Mining \& Technology, Beijing, \\ 100083, China \\ ${ }^{2}$ PetroChina Qinghai well logging Business department, Haixi, 817000, China \\ aemail: zhangguoying1101@16.com
}

Keywords: Flotation Froth; Speed measurement; Spatial relationship; Tracking

\begin{abstract}
The tracking and speed measurement of flotation froth is important for the control and optimization of flotation industry. A new tracking and speed measurement method based on features and spatial relationship is proposed in the paper. The salient bubble objects are extracted from frames, and they are the tracked and matched objects. These error matching bubbles are filtered by the spatial relationship. The motion speed is calculated from the displacement of the matched bubbles between continuous frames. The experiment result show that the tracking and speed measurement is reliable.
\end{abstract}

\section{Introduction}

The feature analysis of flotation froth video is an important detecting method for the flotation process of the non-ferrous and ferrous metals. The size distribution and motion speed of these froths in image could be used to control the flotation process automatically. The recognizing and tracking method of these froth objects in video is the essential analysis technology of flotation process.

The pixel-based method, the boundary-based method and the region-based methods are the classical image tracking methods [1, 2]. The pixel-based method [3] acquires the velocity of moving objects by analyzing the feature difference of each pixel among different frames, and extracts the pixel information with relatively high computational cost. The boundary-based tracking methods can require the reliable boundary and shape information of objects in image[4,5] The region-based tracking methods aim at finding the region which has the best matching features with that of the detected object in another frame[6].

Features of each object can be combined into a single distance measure for matching. Feature matching method is adaptive for motion tracking of complex scene which consists of dynamic background and synchronized moving objects. Two main approaches of feature extracting exist for the tracking and speed measuring of multi-object video. The first ones are to extract global features from the whole image. While the global features have the advantages of being compact and matching efficiently, but the local and spatial information of image objects are lost. The second ones only focus on the local spatial relationships and objects similarity.

The tracking method proposed in the paper is combined block matching with boundary tracking method. Froth regions are extracting from consecutive image frame, and local features for each froth calculated from froth boundary describing. A froth object is considered being successfully recognized when its features in a frame are almost the same as the features of its tracked object in another frame. 


\section{Froth Features Extracting}

1 Froth boundary describing

Flotation froth image contains a lot of forth with various sizes, tracking froths among frames is the basis of speed measurement. Both froth regions and froth top regions can be used for froth tracking The computational cost of extracting froth region is higher than that of extracting its top region[7,8].

For a given video sequence $\{f(x, y, t), t=0,1,2 \ldots\}$, speed measurement is a challenge problem in the presence of multiple similar moving objects and dynamic background. The bi-level image of flotation froth is composed of froths and background. The boundary of moving froth is important for froth tracking. $O_{i, t}$ denotes the i-th tracked froth in $t$ frame, and $O_{i, t+1}^{\prime}$ denotes its matching froth in $t+1$ frame. $R\left(O_{i, t}\right)$ denotes the pixel set of froth $O_{i, t}, E\left(O_{i, t}\right)$ denotes the pixel set of the closed outer boundary of $O_{i, t}$, and can be automatically obtained from freeman chain code [9,10]. $E\left(O_{i, t}\right)=\left\{p_{1}, p_{2}, \ldots, p_{n}\right\}, \mathrm{n}$ is the number of outer boundary pixels.

2 Froth features

Froth features are composed of gray and shape features, such as area, the height and width, the Centroid of froth [11]. Shape and color features of $O_{i, t}$ are computed from $E\left(O_{i, t}\right)$.

1) The area

Area A of froth $O_{i, t}$ comes from $x$ and $y$ coordinate of each pixel in $E\left(O_{i, t}\right)$, the first pixel is $P_{1}, P_{j}$ and $P_{j+1}$ is adjacent boundary pixel.

$$
\mathrm{A}=\sum_{j=1}^{n-1}\left|\left(p_{j+1, y}-p_{j, y}\right) \times\left(p_{j, x}-p_{1, x}+\frac{p_{j+1, x}-p_{j, x}}{2}\right)\right|
$$

2) The height and width

The height $H$ and width $W$ of froth $O_{i, t}$ comes from the maximum and minimum coordinates of all pixel in $E\left(O_{i, t}\right)$.

$$
\begin{aligned}
& \mathrm{H}=\max \left\{y \mid(\mathrm{x}, \mathrm{y}) \in E\left(O_{i, t}\right)\right\}-\min \left\{y \mid(\mathrm{x}, \mathrm{y}) \in E\left(O_{i, t}\right)\right\} \\
& \mathrm{W}=\max \left\{x \mid(\mathrm{x}, \mathrm{y}) \in E\left(O_{i, t}\right)\right\}-\min \left\{x \mid(\mathrm{x}, \mathrm{y}) \in E\left(O_{i, t}\right)\right\}
\end{aligned}
$$

3) The Centroid

Centroid (Gx, Gy) is located in the center of froth $O_{i, t}$.

$$
\begin{aligned}
& \mathrm{G}_{\mathrm{x}}=\frac{1}{2} \max \left\{y \mid(\mathrm{x}, \mathrm{y}) \in E\left(O_{i, t}\right)\right\}+\frac{1}{2} \min \left\{y \mid(\mathrm{x}, \mathrm{y}) \in E\left(O_{i, t}\right)\right\} \\
& \mathrm{G}_{\mathrm{y}}=\frac{1}{2} \max \left\{x \mid(\mathrm{x}, \mathrm{y}) \in E\left(O_{i, t}\right)\right\}+\frac{1}{2} \min \left\{x \mid(\mathrm{x}, \mathrm{y}) \in E\left(O_{i, t}\right)\right\}
\end{aligned}
$$

4) The average gray value

The average gray value $\bar{I}_{i, t}$ comes from the average gray value of froth $O_{i}$ in $t$ frame, $\bar{I}_{i, t+1}$ is the average gray value of froth $O_{i}$ in $t+1$ frame.

\section{Froth similarity}

The similarity between $O_{i, t}$ in t frame and $O_{i, t+1}^{\prime}$ in $t+1$ frame is computed by a weighted sum of the above four feature. The smaller the relative error of the feature, the larger the feature weight is:

$$
\operatorname{sim}\left(O_{i, t}, O_{i, t+1}^{\prime}\right)=1-\left(w_{1} \frac{\left|A_{i}-A_{i}^{\prime}\right|}{A_{i}}+w_{2} \frac{\left|W_{i}-W_{i}^{\prime}\right|}{W_{i}}+w_{3} \frac{\left|H_{i}-H_{i}^{\prime}\right|}{H_{i}}+w_{4} \frac{\left|\bar{I}_{i}-\bar{I}_{i}^{\prime}\right|}{\bar{I}_{i}}\right)
$$

The i-th feature weight $w_{i}$ is based on the relative error between feature $f_{i}$ of $O_{i, t}$ and $f_{i}^{\prime}$ of $O_{i, t+1}^{\prime}$. The larger the relative error is, the smaller the weight is. The sum of all features weight is equal to 1.

$$
w_{\mathrm{i}}=\frac{1}{n-1}-\frac{\left|f_{i}-f_{i}^{\prime}\right| / f_{i}}{(n-1) \sum_{i=1}^{n}\left(\left|f_{i}-f_{i}^{\prime}\right| / f_{i}\right)}
$$


Such as the weight of area feature is in the following.

$$
w_{1}=\frac{1}{3}-\frac{\frac{\left|A_{i}-A_{i}^{\prime}\right|}{A_{i}}}{3\left(\frac{\left|A_{i}-A_{i}^{\prime}\right|}{A_{i}}+\frac{\left|W_{i}-W_{i}^{\prime}\right|}{W_{i}}+\frac{\left|H_{i}-H_{i}^{\prime}\right|}{H_{i}}+\frac{\left|\bar{I}_{i}-\bar{I}_{i}^{\prime}\right|}{\bar{I}_{i}}\right)}
$$

If the similarity of two froths located in two consecutive frame is greater than threshold, the two froths are the same tracked froth.

\section{Design FrothTracking and Checking}

1 Froth tracking

Features matching is the most widely used method in motion tracking. Instead of tracking all froths, the motion of salient froths can represent the motion of scene. A predefined froth ordering is necessary for froth matching. Sorting salient objects in t frame according to the area feature. The object $O_{1, t}$ with the largest area is tracked firstly, the possible matching froth $O_{1, t+1}^{\prime}$ in $t+1$ frame should have the largest similarity with $O_{1, t}$.

The motion of the first tracked froth $O_{1, t}$ can be used to estimate the new location of other froths after moving. An adaptive window based on the location of $O_{1, t}$ is defined in $t+1$ frame and used to track the motion of $O_{1, t}$. The size of window is almost four times the size of froth. The matching froth $O_{1, t+1}$ can be detected out rapidly within the window. The width $W_{w}$ and height $W_{w}$ of window is:

$$
\begin{aligned}
& W_{w}=4 W\left(O_{i, t}\right)+\left|G_{x}\left(O_{1, t+1}^{\prime}\right)-G_{x}\left(O_{1, t}\right)\right| \\
& H_{w}=4 H\left(O_{i, t}\right)+\left|G_{y}\left(O_{1, t+1}^{\prime}\right)-G_{y}\left(O_{1, t}\right)\right|
\end{aligned}
$$

A new multi-object tracking and speed measurement method based on spatial relationship of froths is proposed. How to search the matching froth $O_{1, t+1}^{\prime}$ in $t+1$ frame which has the almost same features as the tracked $O_{i, t}$ in $t$ frame is the key of froth tracking.

\section{Froth checking}

A lot of froths have on similar color and shape. Checking in pairs can guarantee the correctness of froth matching, $O_{1, t+1}^{\prime}$ and $O_{i+1, t+1}^{\prime}$ are matched with $O_{i, t}$ and $O_{i+1, t}$ respectively. The space elationship of $O_{i, t}$ and $O_{i+1, t}$ in $t$ image must be consistent with that of matching froth $O_{i+1, t+1}^{\prime}$ and $O_{i+1, t+1}^{\prime}$ in $t+1$ image. $x$ coordinate difference between froth $O_{i, t}$ and $O_{i+1, t}$ in $t$ frame should be checked with that of $O_{i, t+1}^{\prime}$ and $O_{i+1, t+1}^{\prime}$ in $t+1$ frame by formula 8 . So is the $y$ coordinate difference. The checking formula is defines as the following formula.

$$
\begin{aligned}
& \left|\left(G_{x}\left(O_{i, t}\right)-G_{x}\left(O_{i+1, t}\right)\right)-\left(G_{x}\left(O_{i, t+1}^{\prime}\right)-G_{x}\left(O_{i+1, t+1}^{\prime}\right)\right)\right|<\varepsilon \\
& \left|\left(G_{y}\left(O_{i, t}\right)-G_{y}\left(O_{i+1, t}\right)\right)-\left(G_{y}\left(O_{i, t+1}^{\prime}\right)-G_{y}\left(O_{i+1, t+1}^{\prime}\right)\right)\right|<\varepsilon
\end{aligned}
$$

If $O_{i, t}$ and $O_{i+1, t}$ is not checked successful, their tracking must be discarded, and new froths should be tracked and checking continuously.

After $m$ froths are checked successfully, moving speed of video are estimated based on the time interval between two frames by formula 9 .

$$
\begin{aligned}
& V_{x}=\frac{1}{m \Delta t} \sum_{i, j=1}^{m}\left(G_{x}\left(O_{i, t}\right)-G_{x}\left(O_{i, t}^{\prime}\right)\right) \\
& V_{y}=\frac{1}{m \Delta t} \sum_{i, j=1}^{m}\left(G_{y}\left(O_{i, t}\right)-G_{y}\left(O_{i, t}^{\prime}\right)\right)
\end{aligned}
$$




\section{Experimental Results and Analysis}

The method in this letter is validated by flotation video, and two consecutive frames are shown in Fig.1(a) and Fig.1(b) which include a lots of froths with similar color and shape. The bi-level image of Fig.1(a) and Fig.1(b) are shown in Fig.2(a) and Fig.2(b) respectively. These central regions of froths are high lightness, and tracking highlight regions rather than entire froths can avoid image segmentation and improve tracking efficiency.

Fig.1(a) and Fig.1(b) are two consecutive froth frames with size of 516×389, Fig.2(a) and Fig.2(b) are bi-level images of Fig.1(a) and Fig.1(b).

These froths whose area is less than 20 pixels in Fig.2(a) and Fig.2(b) are filtered out by de-noising operation, the salient six large froths are marked in Fig.3(a) and Fig.3(b).

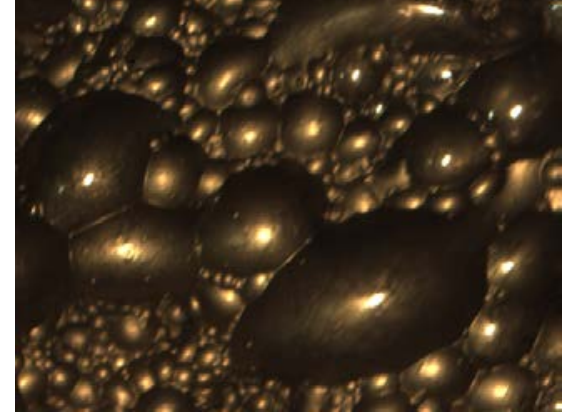

(a) The first frame

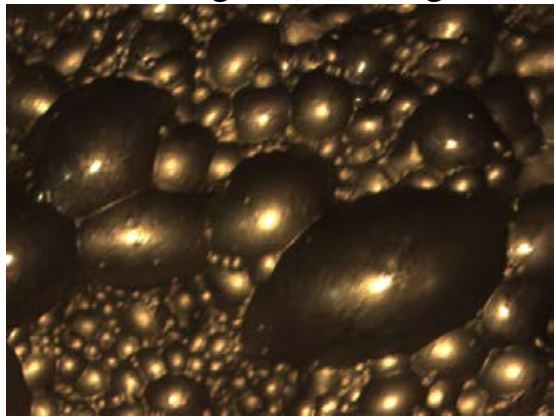

(b) The second frame

Fig.1. Two adjacent frames

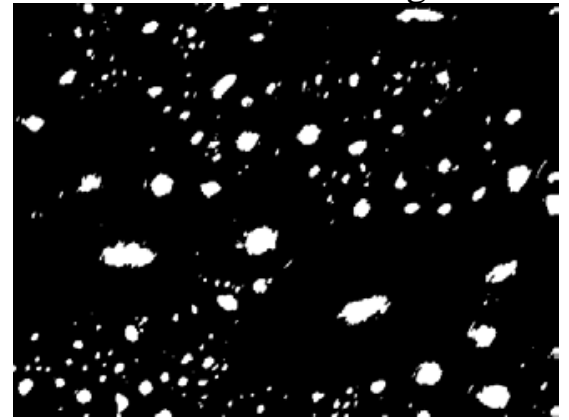

(a) The binary image of Fig.1(a)

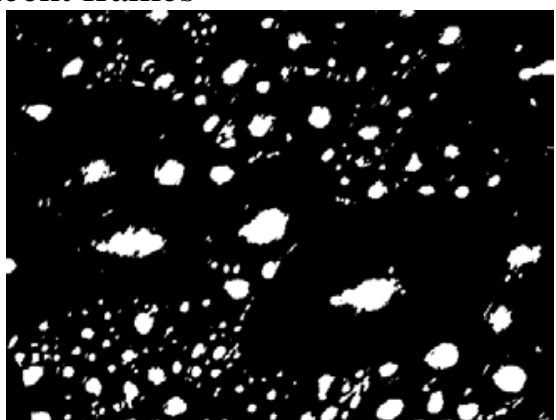

(b) The binary image of Fig.1(b)

Fig.2. Binary image of two adjacent frames

The shape features of six froths are shown in table 1. All of the similarities of six froths between Fig.3(a) and Fig.3(b) are large than threshold 0.6. Six froths are matched successfully and the same froth between Fig.3(a) and between Fig.3(b) are marked by the same color.

Table 1 Shape features of froths

\begin{tabular}{|l|c|c|c|c|c|c|c|c|}
\hline \multirow{2}{*}{ Froth } & \multicolumn{2}{|c|}{$\left(\mathrm{G}_{\mathrm{x}}, \mathrm{G}_{\mathrm{y}}\right)$} & \multicolumn{2}{c|}{ Area } & \multicolumn{2}{c|}{ Width } & \multicolumn{2}{c|}{ Height } \\
\cline { 2 - 9 } & $\mathrm{t}$ & $\mathrm{t}+1$ & $\mathrm{t}$ & $\mathrm{t}+1$ & $\mathrm{t}$ & $\mathrm{t}+1$ & $\mathrm{t}$ & $\mathrm{t}+1$ \\
\hline Green & 108,236 & 117,218 & 841 & 1097 & 53 & 50 & 25 & 28 \\
\hline Red & 331,289 & 338,269 & 733 & 1193 & 65 & 66 & 29 & 31 \\
\hline cyan & 229,223 & 242,204 & 633 & 1017 & 39 & 46 & 27 & 34 \\
\hline Yellow & 453,369 & 473,347 & 509 & 595 & 31 & 31 & 21 & 25 \\
\hline Pink & 393,348 & 409,326 & 395 & 565 & 24 & 31 & 20 & 24 \\
\hline Blue & 384,12 & 153,153 & 386 & 471 & 40 & 27 & 13 & 23 \\
\hline
\end{tabular}


Table 2 The checked results of the tracked objects

\begin{tabular}{|c|c|c|c|c|c|c|}
\hline Froth & Sim & $\Delta \mathrm{x}$ & $\Delta \mathrm{y}$ & Check & $v_{x}$ & $v_{y}$ \\
\hline Green & 0.887 & \multirow{2}{*}{2} & \multirow{2}{*}{2} & \multirow{2}{*}{$\mathrm{Y}$} & 9 & -16 \\
\hline Red & 0.873 & & & & 7 & -16 \\
\hline Cyan & 0.767 & \multirow{2}{*}{7} & \multirow{2}{*}{3} & \multirow{2}{*}{$\mathrm{Y}$} & 13 & -19 \\
\hline Yellow & 0.899 & & & & 20 & -21 \\
\hline Pink & 0.774 & \multirow{2}{*}{347} & \multirow{2}{*}{$\begin{array}{c}16 \\
3\end{array}$} & \multirow{2}{*}{$\mathrm{N}$} & - & - \\
\hline Blue & 0.686 & & & & - & - \\
\hline
\end{tabular}

Red and green froths is verified successfully by formula 7 and shown in table 2. Red and green froths in Fig.4(a) are successfully checked with that in Fig.4(b). The x distance difference of red and green froths between Fig.4(a) and Fig.4(b) is 2 pixels, so is the y difference. The checked results of six objects are shown in table 2.

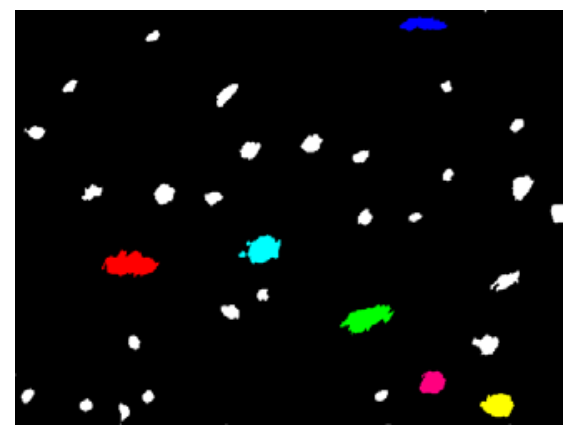

(a) Extracted large objects in Fig.1(a)

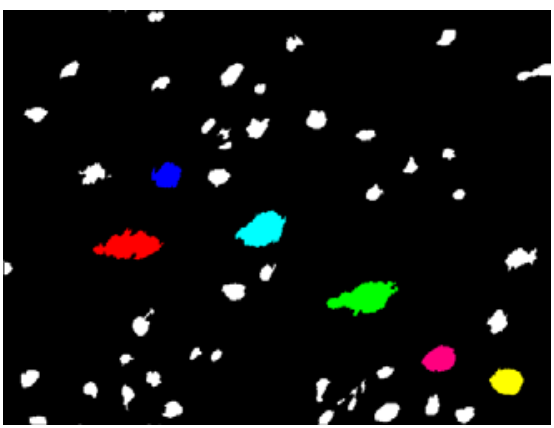

(b) Extracted large objects in Fig.1(b)

Fig.3. Extracted large objects in Fig.1(a) and Fig.1(b)

Fig.3(a) and Fig.3(b) are large objects extracting images of Fig.1(a) and Fig.1(b) after de-noising. Fig.4(a) marks the 1-th and 2-th tracked froth in Fig.1(a), Fig.4(b) marks the two matched froths in Fig.1(b).

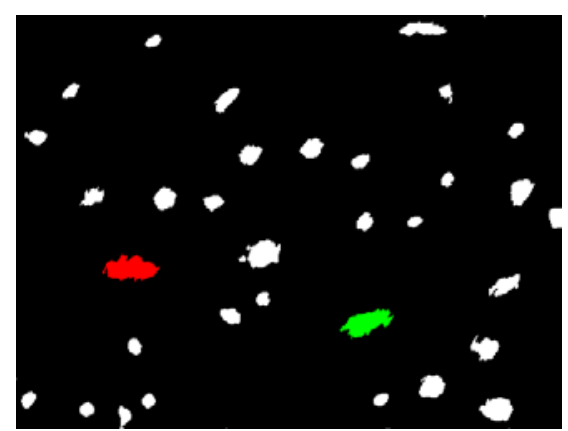

(a) The 1-th and 2-th tracked froth in Fig.1(a)

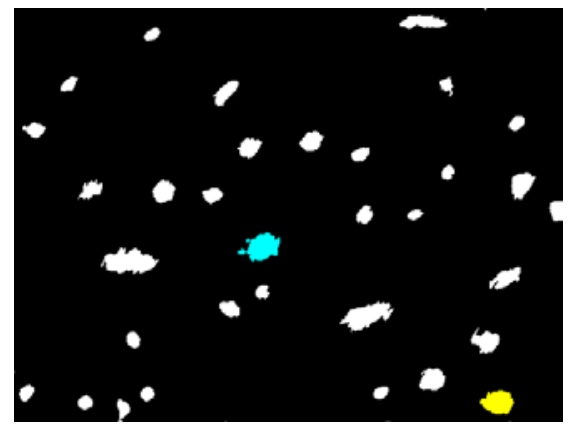

(c) The 3-th and 4-th tracked froth in Fig.1(a)

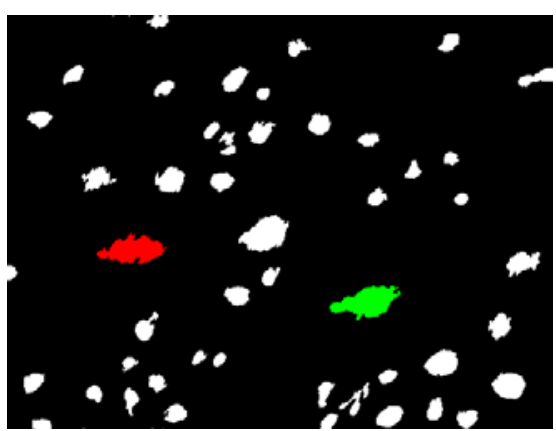

(b) The 1-th and 2-th tracked froth in Fig.1(b)

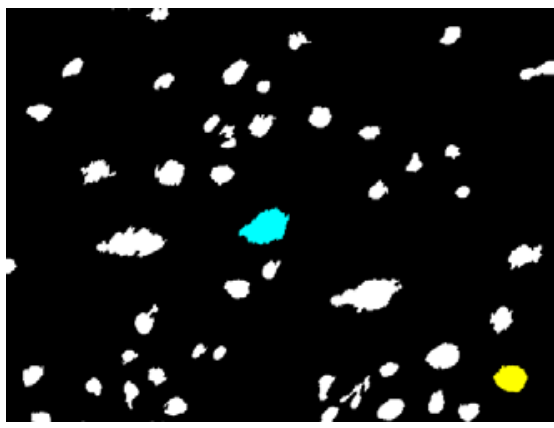

(d) The 3-th and 4-th tracked froth in Fig.1(b) 


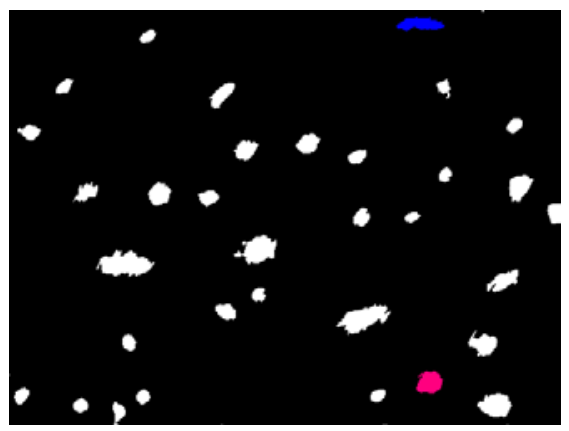

(e) the 5-th and 6-th tracked froth in Fig.1(a)

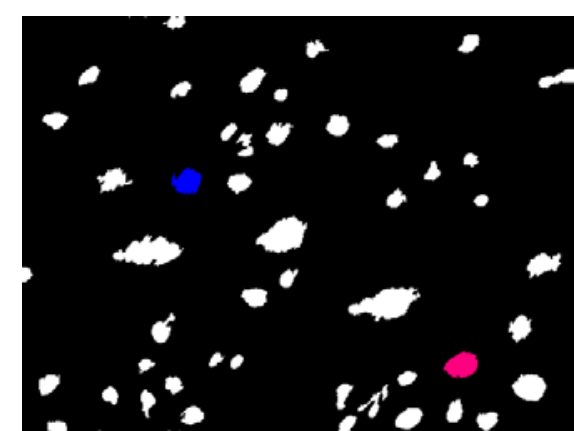

(f) the 5-th and 6-th tracked froth in Fig.1(b)

Fig.4. The couple tracked froths in Fig.1(a) and Fig.1(b)

Fig.4(c) marks the 3-th and 4-th tracked froth in Fig.1(a), Fig.4(d) marks the matched froths in Fig.1(b). Fig.4(e) marks the the 5-th and 6-th tracked froth in Fig.1(a), Fig.4(f) marks the matched froths in Fig.1(b).

\section{Conclusion}

We propose a tracking and speed measurement algorithm which combines similarity comparison and spatial relationship checking.

1) Extracting froth features including color and shape features is set up. Froth similarity between consecutive frames is compared.

2) Matching and checking froths in pair can ensure tracking accuracy.

The performance of the proposed algorithm for multi-object tracking and speed measurement in video sequence is excellent.

\section{Acknowledgement}

In this paper, the research was sponsored by The national high technology research and development plans (863 plan) (No.2013AA064303)

\section{References}

[1] J. J. Francis and G. de Jager. An investigation into the suitability of various motion estimation algorithms for froth imaging. In Proceedings of the 1998 South African Symposium on Communications and Signal Processing - COMSIG'98, Rondebosch, South Africa, 1998, pp: 139 -142 ,

[2] J. J. Francis, G. de Jager. Watershed segmentation based motion estimation of froth image sequences. In Proceedings of the Ninth Annual South African Workshop on Pattern Recognition, Stellenbosch, South Africa, 1998,pp: $44-49$.

[3] K. K. Nguyen. Flotation Froth Characterisation By Using Vision Technology. PhD thesis, University of Queensland, 1998.

[4] Wang R B, Yu T H, Jia Y. Study of stone detection method based on edge extraction and track. J of Computer Applications, 2006 01, pp. 114-119(in Chinese).

[5] Y. K. Liu, Borut Žalik. An efficient chain code with Huffman coding. Pattern Recognition, 2005, 38, pp:553-557

[6] Kim, J.B., Kim, H.J. Efficient region-based motion segmentation for a video monitoring system. Pattern Recognition Letter, 2003, 24 ,pp:1-3

[7] Zhang G.Y., Zhu H., Xu n. Flotation bubble image segmentation based on seed region boundary growing. Mining Science and Technology (China). 21,2011,pp: 239-242 . 
[8] G. Y. Zhang, Q. l. Feng, C. J. Mu. Froth Image Segmentation Based on Adaptive Ray Cluster. 2010 IEEE International conferrence on nNetworking, Sensing and Control, Chicago, USA. 298-301.

[9] Freeman, H. On the encoding of arbitrary geometric configurations. IEEE Trans. Electron. Comput., 1961, 10, pp: 260-268

[10] Hermilo S.C., Ernesto B., Ramón M. Efficiency of chain codes to represent binary objects, Pattern Recognition, 2007, 40, pp:1660-1674

[11] Gordon Forbes. Texture and Bubble Size Measurements for Modeling Concentrate Grade in Flotation Froth Systems[D], University of Cape Town, 2007. 